\title{
A Case of Superior Canal Dehiscence Treated by Resurfacing the Superior Canal Defect via the Transmastoid Approach
}

\author{
Mitsuo Sato ${ }^{1)}$, Takaaki Kobayashi ${ }^{1)}$, Kazuya Saito ${ }^{1)}$, \\ Takao Imai ${ }^{2)}$ and Katsumi Doi ${ }^{1)}$
}

\begin{abstract}
In this study, we report on a patient who had superior canal dehiscence and was treated by resurfacing the defect via the transmastoid approach. An 80-year-old male patient had the chief complaint of vertigo when he touched his left porus acusticus externus. As leftward nystagmus was induced by change in middle ear pressure and the existence of superior canal dehiscence was confirmed with computed tomographic scans (CT) of the temporal bones, we suspected an idiopathic labyrinthine fistula similar to the superior canal dehiscence syndrome (SCDS). At first, he was treated with medication, but as his vertigo was getting worse, he underwent surgery. A wide mastoidectomy was performed identifying the middle fossa dura. The superior semicircular canal was exposed together with the angle between the superior semicircular canal and the middle fossa bone. A bone pate was insinuated down the dehiscent canal. After the operation, no nystagmus was induced by change in the middle ear pressure and the patient did not feel any vertigo. When we anticipate the possibility of a transmastoid approach to a defect of superior canal based on preoperative CT findings, we should select this approach because the approach can avoid the more invasive middle fossa craniotomy.
\end{abstract}

Keywords : transmastoid approach, vertigo, superior canal dehiscence syndrome, resurface the canal defect

\section{References}

1) Minor LB, Solomon D, Zinreich JS, et al. : Sound- and/or pressure-induced vertigo due to bone dehiscence of the superior semicircular canal. Arch Otolaryngol Head Neck Surg 124: 249-258, 1998.

2) Imai T, Takeda N, Morita M, et al. : Rotation vector analysis of eye movement in three dimensions with an infrared CCD camera. Acta Otolaryngol 119: 24-28, 1999.

3) Ishijima K : Superior and posterior canal dehiscence syndrome. Pract Otol (Kyoto) 100: 962-963, 2007.

4) Minor LB : Clinical manifestations of superior semicircular canal dehiscence. Laryngoscope 115: 1717-1727, 2005.

5) Merchant SN and Rosowski JJ : Conductive hearing loss caused by third-window lesions of the inner ear. Otol Neurotol 29: 282-289, 2008.
6) Minor LB, Carey JP, Cremer PD, et al. : Dehiscence of bone overlying the superior canal as a cause of apparent conductive hearing loss. Otol Neurotol 24: 270-278, 2003.

7) Brantberg K, Bergenius J and Tribukait A : Vestibular-evoked myogenic potentials in patients with dehiscence of the superior semicircular canal. Acta Otolaryngol 119: 633-640, 1999.

8) Crovetto M, Areitio E, Elexpuru J, et al. : Transmastoid approach for resurfacing of Superior Semicircular Canal dehiscence. Auris Nasus Larynx 35: 247-249, 2008.

9) Fiorino F, Barbieri F, Pizzini FB, et al. : A dehiscent superior semicircular canal may be plugged and resurfaced via the transmastoid route. Otol Neurotol 31: 136-139, 2010.

10) Friedland DR and Michel MA : Cranial thickness in superior canal dehiscence syndrome: implications for canal resurfacing surgery. Otol Nuerotol 27: 346-354, 2006.
1) Department of Otolaryngology, Kinki University Medical School

2) Department of Otorhinolaryngology, Head-Neck Surgery, Osaka University Graduate School of Medicine
Corresponding Author Address : Mitsuo Sato qqd755pd@spice.ocn.ne.jp 


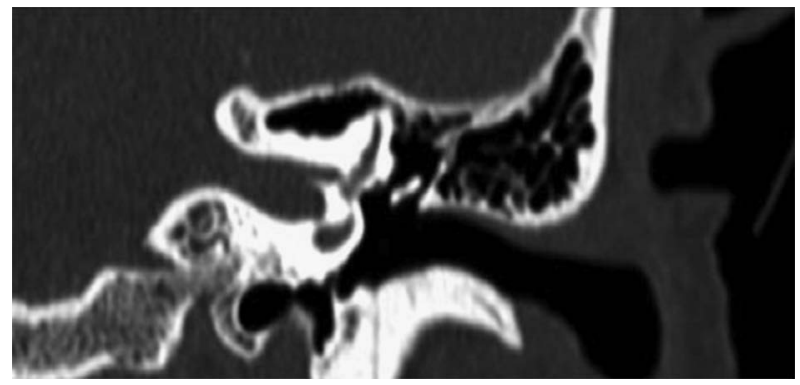

A coronal CT image showing bone dehiscence between the superior semicircular canal and the middle ear

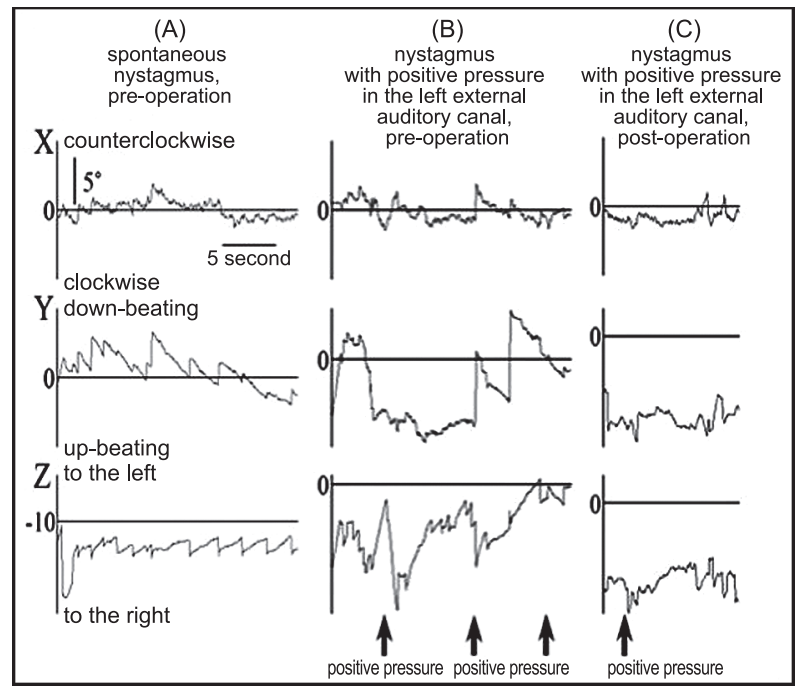

Rotation vector analysis of eye movement in three dimensions with an infrared CCD camera

A: Pre-operation, mild rotatory nystagmus to the right is observed

B: Pre-operation with positive pressure in the external auditory canal, horizontal eye movement to the left is seen

C: Six months after the operation with positive pressure in the external auditory canal, the spontaneous nystagmus has disappeared 